\title{
Low-frequency electromagnetic waves and instabilities within the Martian bi-ion plasma
}

\author{
K. Sauer ${ }^{1,4}$, E. Dubinin ${ }^{1,2,4}$, K. Baumgärtel ${ }^{3,4}$, and V. Tarasov Ban $^{2,4}$ \\ ${ }^{1}$ Max-Planck-Institut für Aeronomie, 37191 Katlenburg-Lindau, Germany \\ ${ }^{2}$ Space Research Institute, 117810 Moscow, Russia \\ ${ }^{3}$ Astrophysikalisches Institut Potsdam, 14437 Potsdam, Germany \\ ${ }^{4}$ International Space Science Institute (ISSI), 3012 Bern, Switzerland
}

(Received September 29, 1997; Accepted January 28, 1998)

\begin{abstract}
The Martian environment is characterized by the presence of heavy (oxygen) ions of planetary origin which strongly influence the solar wind dynamics, including the bow shock structure and position and may cause additional plasma boundaries in the magnetosheath. In this paper the dispersion characteristics of low-frequency electromagnetic waves (LFEW) in the proton gyrofrequency range are studied. The excitation of these waves results from the relative motion between the solar wind protons and planetary heavy ions, which are considered as unmagnetized and, therefore, may act like a beam in the solar wind. The model takes into account the small extension of the Martian magnetosphere compared with the pickup gyroradius of an exospheric ion. From the dispersion analysis it was found that the most unstable waves with relatively high growth rates propagate oblique to the ambient magnetic field. For small propagation angle to the magnetic field these are right-hand polarized whistler waves in the solar wind frame, and due to Doppler shift they appear near to the proton cyclotron frequency as left-hand polarized waves in the beam (spacecraft) frame. We suggest that the sporadic LFEW emission as seen in the upstream region of Mars by Phobos-2 may indicate the existence of localized "heavy ion bunches" whose origin is relatively unclear, but a possible relation to the Martian moons cannot be excluded. Especially, the socalled Phobos events marked by spectral peaks around the proton cyclotron frequency may be interpreted as signatures of the solar wind interaction with a tenuous gas torus. A comparable situation is known from the AMPTE $\mathrm{Ba}$ and $\mathrm{Li}$ releases where during the late stages of the experiments an enhanced proton cyclotron emission was observed as well. Another important aspect of LFEW excitation is its role in proton deceleration and heating upstream the bow shock where turbulent processes may provide a strong momentum coupling between the solar wind and the newly generated ions of planetary origin.
\end{abstract}

\section{Introduction}

Among the planets, Mars is unique in that it has neither an intrinsic magnetic field (like Earth) nor has a dense ionosphere (like Venus). However, unlike the Moon which simply absorbs the solar wind particles on its surface, the Martian ionosphere/exosphere establishes an obstacle to the solar wind which gives rise to the formation of a bow shock (e.g. Riedler et al., 1989). Since observational evidence for its existence was provided by the first space missions to Mars, the question remained as to what mechanism is responsible for its formation. In contrast to planets where an ionopause or magnetopause causes the bow shock, Mars resembles more rather a weak comet where mass loading essentially contributes to the shock formation. This is supported by the data provided by the Phobos-Mission in 1989. The obstacle boundary seems to be an ion composition boundary which separates the solar wind from a plasma of mainly planetary origin (Sauer et al., 1994, 1997a; Dubinin et al., 1996). But, many questions are still open which concern the physical processes taking place in the momentum coupling between

Copy right () The Society of Geomagnetism and Earth, Planetary and Space Sciences (SGEPSS); The Seismological Society of Japan; The Volcanological Society of Japan; The Geodetic Society of Japan; The Japanese Society for Planetary Sciences. the solar wind protons and heavy planetary ions for the specific situation in what the interaction region is much smaller than the pick-up gyroradius. Using1D hybrid code simulations of solar wind massloading (Omidi and Winske, 1987) at comets, it has been proven one decade ago that the excitation of low-frequency electromagnetic waves (LFEW) and their growth to large amplitudes and subsequent nonlinear evolution play a crucial role in plasma deceleration and heating. Subsequently, 3D hybrid code simulations have been developed for Mars (Brecht, 1997), and they are a very useful steps in improving our picture about the complex plasma environment of this planet. Unfortunately, the present models suffer from the fact that no ionospheric/ exospheric interaction have been included.

During the last years the role of minor heavy ions has been increasingly recognized, for the solar wind itself to explain the different proton/alpha particle behavior with respect to acceleration and heating. A number of electromagnetic instabilities were considered which are triggered by ion beams parallel to the magnetic field (Gomberoff et al., 1996, and references therein). Another related topic concerns waves generated by gyroresonant pickup ions in the vicinity of comets (Tsurutani and Smith, 1986), and very recently minor ion cyclotron waves have been observed at Galileos's 
Io encounter (Warnecke et al., 1997; Huddleston et al., 1997). The purpose of this paper is to show that planetary ions in the vicinity of Mars may even lead to strong low-frequency electromagnetic wave excitation by beam-plasma interaction in the solar wind, but under much different conditions. For Mars and other similar situations, such as less active comets and possibly Pluto (Sauer et al., 1997b), where the interaction region is much smaller than the heavy ion pickup radius, the newly generated ions can be considered as unmagnetized, regardless of the magnetic field orientation. We will show that this ion beam may act as an effective source of low-frequency wave excitation in two branches: in the beam frame, one is near to the proton cyclotron frequency and the other far below of that. Both exhibit their maximum growth away from parallel propagation to the magnetic field.

Our analysis is based on the general dispersion theory for a cold bi-ion plasma, in which the (heavy) beam ions are treated as unmagnetized. Since we restrict ourself to lowfrequency waves (below the lower hybrid frequency), the electrons are taken as massless. An alternative approach using the bi-ion fluid approach (Sauer et al., 1994, 1996) is described in the paper by Baumgärtel et al. (1998) in which special emphasis is given to the so-called Phobos events. They are interpreted as LFEW signatures of the solar wind interaction with a gas torus produced by the Martian moon Phobos.

The layout of this paper is as follows. Section 2 describes examples of low-frequency electro-magnetic waves under conditions which we believe are relevant for our studies: 2.1
Localized LFEW emissions near the crossing of the Phobos2 spacecraft with orbit of the Martian moon Phobos (socalled Phobos events). It is suggested that these emissions give evidence of a heavy ion source (gas torus) along the Phobos orbit. 2.2 Strong deceleration of the solar wind upstream of the Martian bow shock. Simultaneous observation of proton cyclotron waves are seen as hints of their role in momentum coupling between the solar wind and the exospheric plasma. 2.3 Proton cyclotron waves during the late stages of the AMPTE Ba and Li releases. The observed spectral peaks near the proton cyclotron frequency seem to be a convincing observation of LFEW excitation by minor heavy ion beam-plasma interaction. In Section 3 we derive the dispersion relation of low-frequency electromagnetic waves in a cold plasma composed of protons and heavy, unmagnetized ions moving relative to the protons regardless of the magnetic field orientation. Results of the dispersion analysis are shown. Section 4 contains a discussion with a outlook to future work which requires a kinetic treatment of the considered instability.

\section{Possible Examples of Beam Excited LFEWs}

We describe three examples where electromagnetic wave emission near the proton cyclotron frequency was observed under conditions which may correspond to a heavy ion beam-plasma interaction.

\subsection{The Phobos events}

Significant plasma and magnetic field perturbations were observed by the Phobos-2 spacecraft near the crossings of the orbit of the Martian moon Phobos. Figure 1 shows the

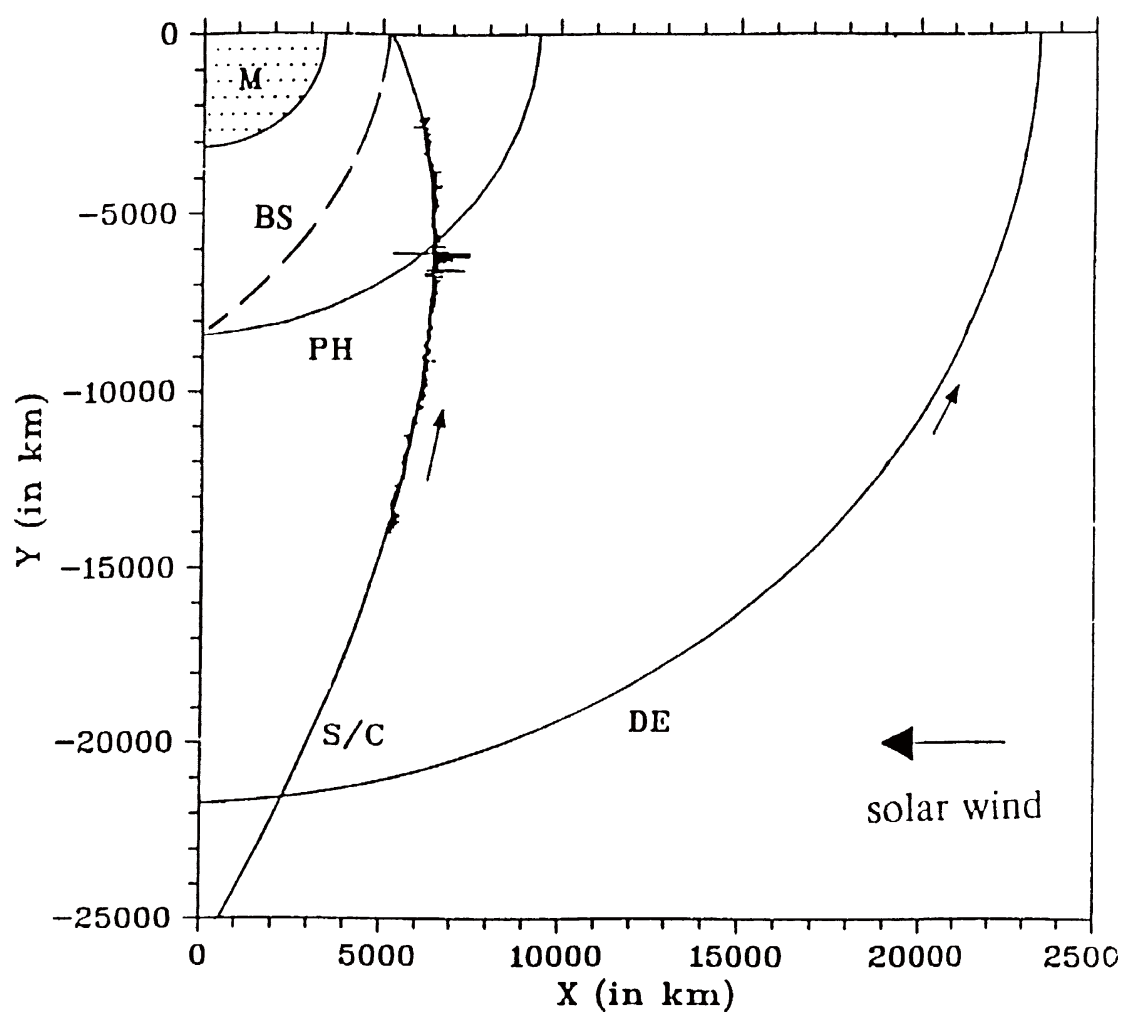

Fig. 1. "Phobos event" of the third elliptical orbit on Feb. 8, 1989. The deviation of the magnetic field magnitude B from its average value is attached to the spacecraft trajectory indicating the event at the Phobos orbit crossing. The orbits of the moons Phobos (PH) and Deimos (DE) and the spacecraft trajectory (S/C) are projected onto the ecliptic $x-y$ plane. The bow shock (BS) is marked by the dashed curve. 
(relative) magnetic field variation along the spacecraft orbit for the third elliptical orbit on February 8, 1989. For the same orbit, the magnetic field magnitude is plotted in Fig. 2(a) together with the low-frequency magnetic spectrum as result of a wavelet analysis (Tarasov et al., 1998). Just at the time (UT $\approx 04: 55$ ) where the "Phobos event" is seen as a strong magnetic field perturbation, a significant wave emission occurs near the proton cyclotron frequency $\Omega_{\mathrm{p}}$ (traced by a red line). Another maximum at much lower frequencies $\left(\geq 0.1 \Omega_{p}\right)$ seems to be a significant feature as well. The idea is that the observed event results from the interaction of the solar wind with a torus plasma population which is continually being replenished by the ionization of neutral particles from Phobos (Dubinin et al., 1990; Dubinin, 1993; see also Sauer et al., 1993, 1995; Baumgärtel et al., 1996). More details are given in the paper by Baumgärtel et al. (1998).

PHOBOS-2

FEBRUARY 8, 1989
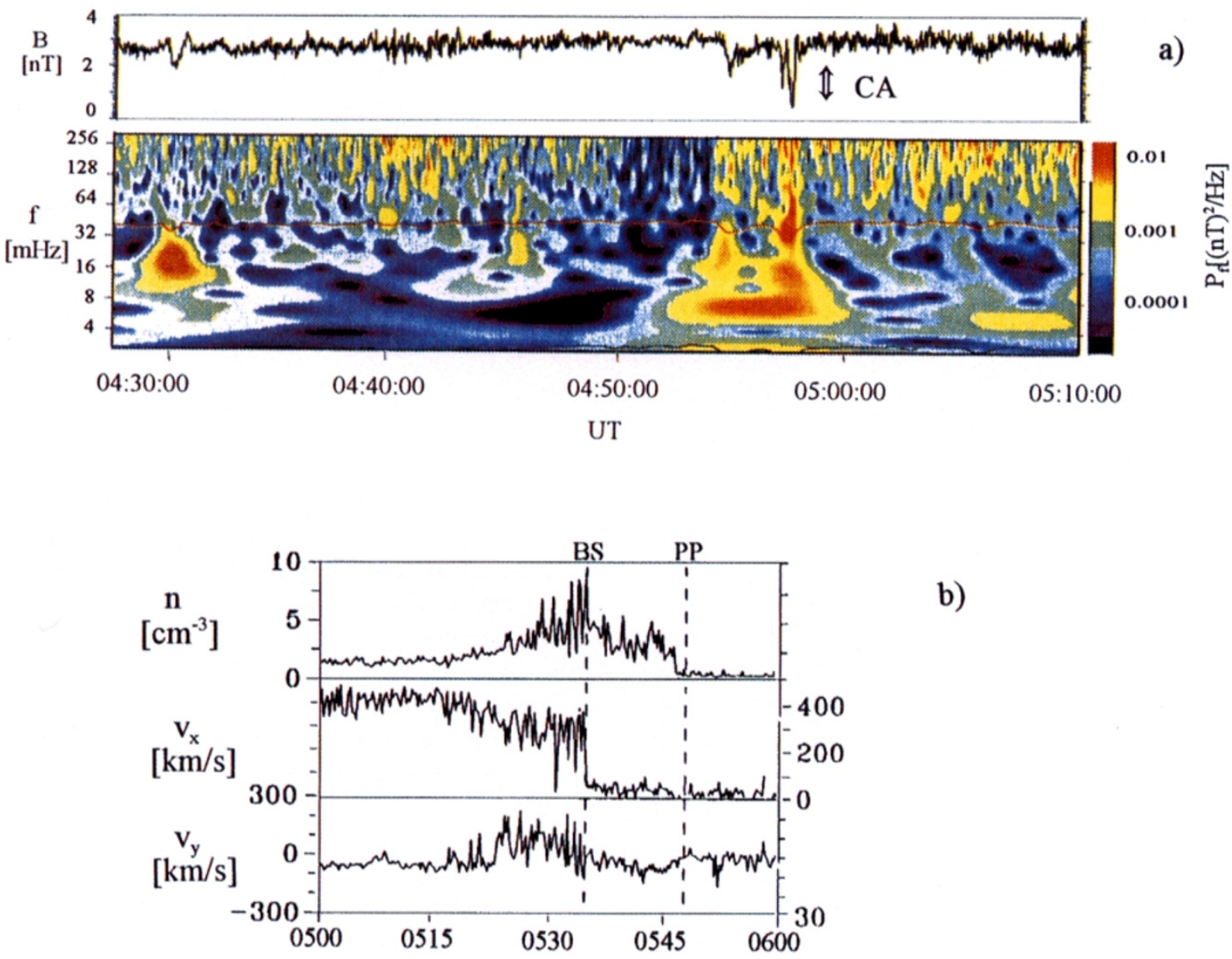

b)

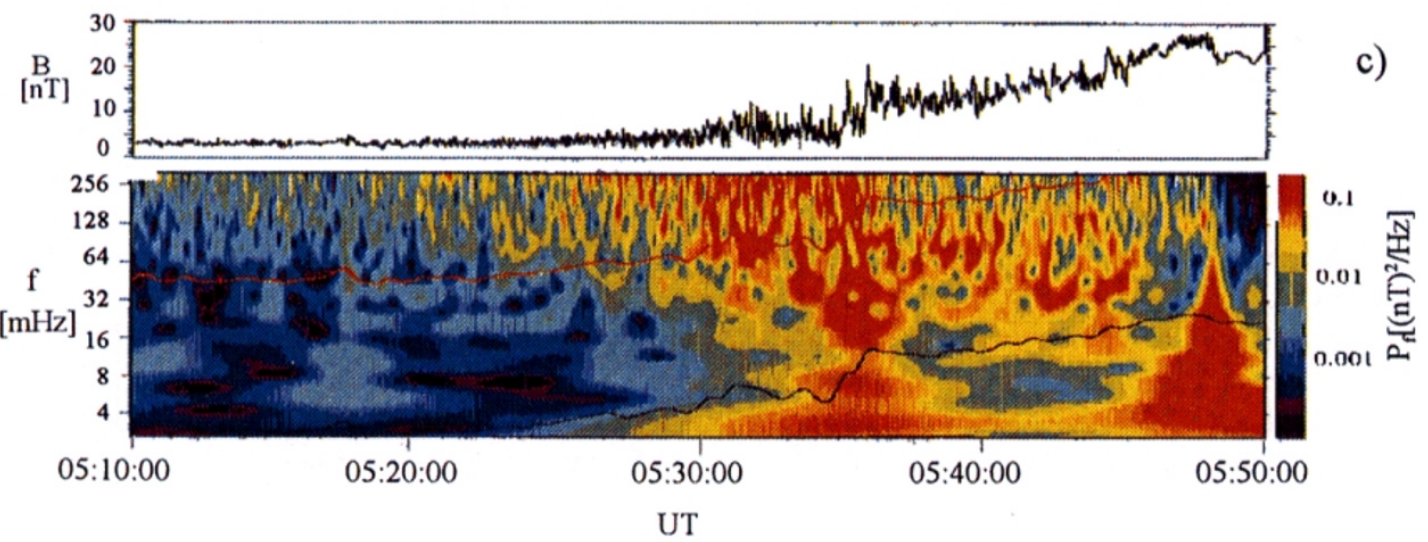

Fig. 2. (a) "Phobos event" of the third elliptical orbit on Feb. 8, 1989. The upper panel shows the FGMM magnetic field data. The event is marked by strong variations just before the Phobos orbit is crossed. Closest approach is indicated by the arrow. The bottom panel represents the lowfrequency magnetic field power as result of wavelet analysis. The cyclotron frequency $\Omega_{\mathrm{p}}$ is traced by a red line. (b) Deceleration of the solar wind upstream the Martian bow shock. From top to bottom: Proton density $n$ and velocity components $\mathrm{v}_{x}$, $\mathrm{v}_{y}$ from ASPERA during the third elliptical orbit on Feb. 8, 1989 (adapted from Sauer et al., 1992). (c) Low-frequency magnetic field power as result of wavelet analysis in the up- and downstream region of the Martian bow shock (at UT $\approx 05: 36$ ). The proton cyclotron frequency is traced by a red line. 


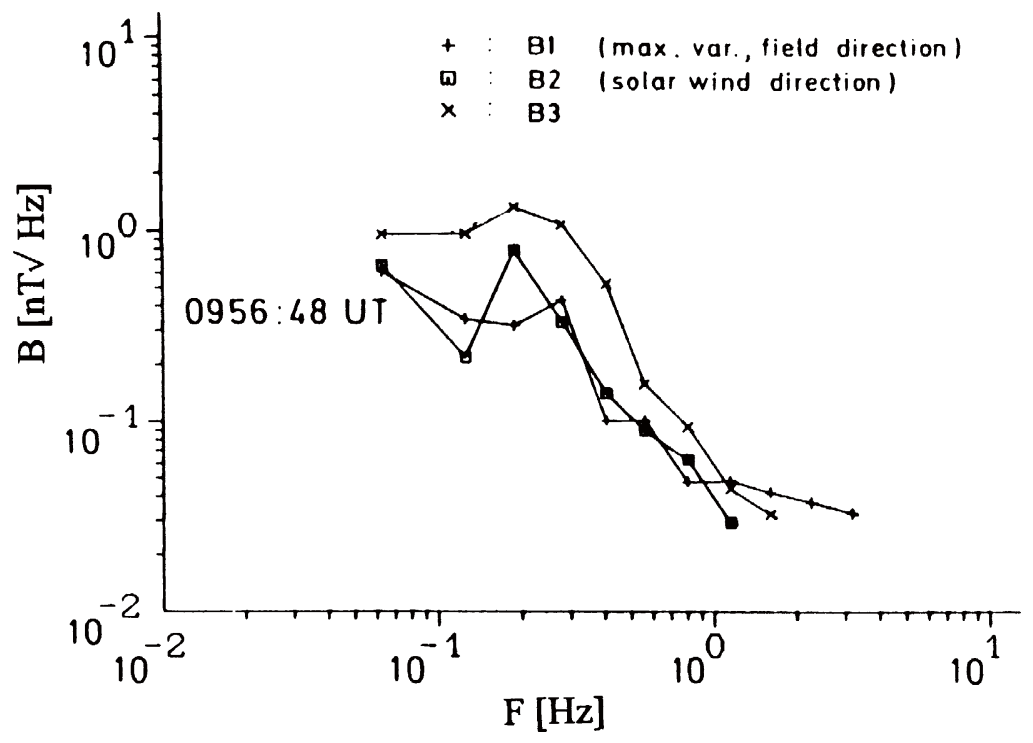

Fig. 3. Spectral density plot from AMPTE lithium release on September 20, 1984 at a late time when the solar wind returned nearly to its undisturbed state (0956:48 UT), adapted from Lühr et al. (1986). A spectral peak at about $0.2 \mathrm{~Hz}$ which is approximately the proton cyclotron frequency for a magnetic field of $10 \mathrm{nT}$ is seen. B1 and B3 denote the directions of maximum and minimum variance, respectively.

\subsection{Solar wind deceleration upstream the Martian bow shock}

The measurements of the proton spectra by the TAUS and ASPERA instruments during the elliptical orbits of the Phobos-2 spacecraft revealed a strong deceleration of nearly $100 \mathrm{~km} / \mathrm{s}$ over around 1,500 km upstream the Martian bow shock (Verigin et al., 1991; Sauer et al., 1992; Dubinin et al., 1994). An example is shown in Fig. 2(b). The essential solar wind deceleration starts at $\approx 0525 \mathrm{UT}$ and continues up to the bow shock at $0535 \mathrm{UT}$. As possible effects the mass-loading of the solar wind by ions originating from the oxygen/ hydrogen corona and/or specularly reflected protons from the bow shock were discussed. But, using stationary massloading models, even with the most favorable parameters for the hot oxygen corona (Ip, 1988) the observed deceleration could not be explained. Perhaps, the envisaged aspect of electromagnetic wave excitation by heavy ion beams may improve our understanding of the most relevant interaction processes. In this context, support comes from the evidence of left-handed elliptically waves at the proton cyclotron frequency during the relevant time period, first reported by Russel et al. (1990). The waves were found to propagate at a small to moderate angle $\left(\leq 30^{\circ}\right)$ to the magnetic field. These earlier results are confirmed by wavelet analysis (Tarasov et al., 1998) shown in Fig. 2(c). An enhanced LFEW emission around the proton cyclotron frequency appears there about 10 min before shock crossing. Russel et al. (1990) suggested that these low-frequency waves are associated with the pickup of newly ionized hydrogen atoms. But, as seen from our subsequent studies, heavy-ion beam excited turbulence seems to be a competitive mechanism for the momentum coupling between the solar wind and planetary ions, in a similar way as it was considered by Papadopoulos et al. (1987) for the AMPTE artificial comet.

\subsection{Proton cyclotron waves at the AMPTE releases}

The different stages of the AMPTE Barium/Li releases
(Gurnett et al., 1986a, b) represent an excellent possibility to test theories of heavy ion-solar wind interaction. Generally, the characteristic dimension of the interaction region for these releases is small (less than about $500 \mathrm{~km}$ ), and so, the assumption of unmagnetized ions, particularly for the central part of the released cloud where the AMPTE/UKS spacecraft instruments made their measurements, seems to be sufficiently justified. In the published literature, evidence for electromagnetic emission near the proton cyclotron frequency was found in the last stage of the second $\mathrm{Li}$ release on September 20, 1986 (Lühr et al., 1986), just before the solar wind relaxes to its undisturbed state (see Fig. 3 ). For a background magnetic field of $10 \mathrm{nT}$, the spectral peak is near $0.2 \mathrm{~Hz}$. The occurrence of this emission particularly in the last stage of the release is thought to be associated with the decrease of the Li cloud density to values below the solar wind density which creates a characteristic situation for weak beam-plasma interaction. Moreover, after re-analyzing the AMPTE/UKS magnetic field data of the Ba release on Dec. 27, 1984 a very pronounced proton cyclotron wave emission over a period of about 200s was found. As a remarkable feature it should be noted, that the beam-excited waves propagate nearly transverse to the ambient magnetic field (magneto-acoustic waves). More details to this topic will be published in a subsequent paper (Sauer et al., 1998).

\section{Dispersion Analysis of LFEW in a Minor Ion Beam-Plasma System}

Our goal is to derive a dispersion relation for low-frequency waves in a bi-ion plasma composed of the solar wind as the core plasma and a minority of unmagnetized heavy ions which are in relative motion to the core and may act as a weak beam. This assumption follows the idea that the size of the interaction region is less than the pick-up gyroradius of the newly created exospheric ions. In such large Larmor 
radius systems a relative streaming of solar wind protons and heavy ions is admissible even transverse to the magnetic field.

We restrict ourselves to a cold plasma of protons (p), heavy ions (h) and massless electrons (e). Their uniform state is characterized by $n_{\mathrm{p}}, n_{\mathrm{h}}, \mathbf{v}_{\mathrm{h}} \neq 0, n_{\mathrm{e}}=n_{\mathrm{p}}+n_{\mathrm{h}}, \boldsymbol{B}$. (To simplify the subsequent algebra, the heavy ion velocity $\mathbf{v}_{\mathrm{h}}$ is taken transverse to the magnetic field $\boldsymbol{B}$.) The appropriate dispersion relation for low-frequency electromagnetic waves (LFEW) can be derived on two ways, either from the general dispersion theory (for example Stix, 1992) or by linearizing the bi-ion Hall-MHD equations used in earlier papers (Sauer et al., 1994, 1996; see also Baumgärtel et al., 1998).

Here, we adopt the general dispersion relation

$$
A N^{4}+B N^{2}+C=0(N=k c / \omega)
$$

to our specific situation: In the limit of massless electrons, the coefficients $A, B$ and $C$ are given by

$$
A=\cos ^{2} \theta, B=-\left(\varepsilon_{x x}+\varepsilon_{y y} \cos ^{2} \theta\right), C=\varepsilon_{x x} \varepsilon_{y y}+\varepsilon_{x y}{ }^{2}
$$

with the dielectric tensor components

$$
\begin{aligned}
& \varepsilon_{x x}=\left[\frac{\omega_{\mathrm{p}}^{2}}{\omega^{2}-\Omega_{\mathrm{p}}^{2}}+\frac{\omega_{\mathrm{h}}^{2}}{\omega^{* 2}}\right], \\
& \varepsilon_{y y}=\left[\frac{\omega_{\mathrm{p}}^{2}}{\omega^{2}-\Omega_{\mathrm{p}}^{2}}+\frac{\omega_{\mathrm{h}}^{2}}{\omega \omega^{*}}\right], \\
& \varepsilon_{x y}=\frac{i}{\omega}\left[\frac{\omega_{\mathrm{p}}^{2} \Omega_{\mathrm{p}}}{\omega^{2}-\Omega_{\mathrm{p}}^{2}}+\frac{\omega_{\mathrm{e}}^{2}}{\Omega_{\mathrm{e}}}\right] .
\end{aligned}
$$

$\omega_{\mathrm{p}}, \omega_{\mathrm{h}}, \omega_{\mathrm{e}}$ are the plasma frequencies of protons, heavy ions and electrons determined by the respective densities $n_{\mathrm{p}}, n_{\mathrm{h}}$ and $n_{\mathrm{e}} . \Omega_{\mathrm{p}}, \Omega_{\mathrm{e}}$ are the corresponding cyclotron frequencies. $\omega^{*}=\omega-\boldsymbol{k} \cdot \mathbf{v}_{\mathrm{h}}$ is the Doppler-shifted frequency in the beam reference frame. $\theta$ is the angle between the magnetic field $\boldsymbol{B}$ (in $z$ direction) and the wave vector $\boldsymbol{k}$ (in the $x$ - $z$ plane). Combination of Eqs. (1)-(5) leads after straightforward algebra to a polynomial of 9th order in the complex variable $\omega=\omega(\boldsymbol{k})$, which is solved by standard numerical procedures.

Figure 4 shows the dispersion of low-frequency electromagnetic waves near the proton cyclotron frequency $\Omega_{p}$ in a cold bi-ion plasma of protons and minor heavy ions for oblique propagation (a: $\theta=30^{\circ}$, b: $\theta=80^{\circ}$ ). Due to the assumption that the heavy ions are unmagnetized, no L (lefthand) mode resonating at the heavy ion cyclotron frequency $\Omega_{\mathrm{h}}$ occurs. But even then, the proton modes have nearly the same characteristics as found in earlier studies without this restriction (e.g. Young et al., 1981): The L mode evolves from the cutoff-frequency (here simply $\left.\omega_{\mathrm{cf}}=n_{\mathrm{h}} /\left(n_{\mathrm{p}}+n_{\mathrm{h}}\right) \Omega_{\mathrm{p}}\right)$, and it becomes coupled to the upper R (right-hand) mode which goes into the whistler branch at higher frequencies. The upper $L$ mode resonates at $\Omega_{\mathrm{p}}$.

As the source of free energy for wave excitation a beam of

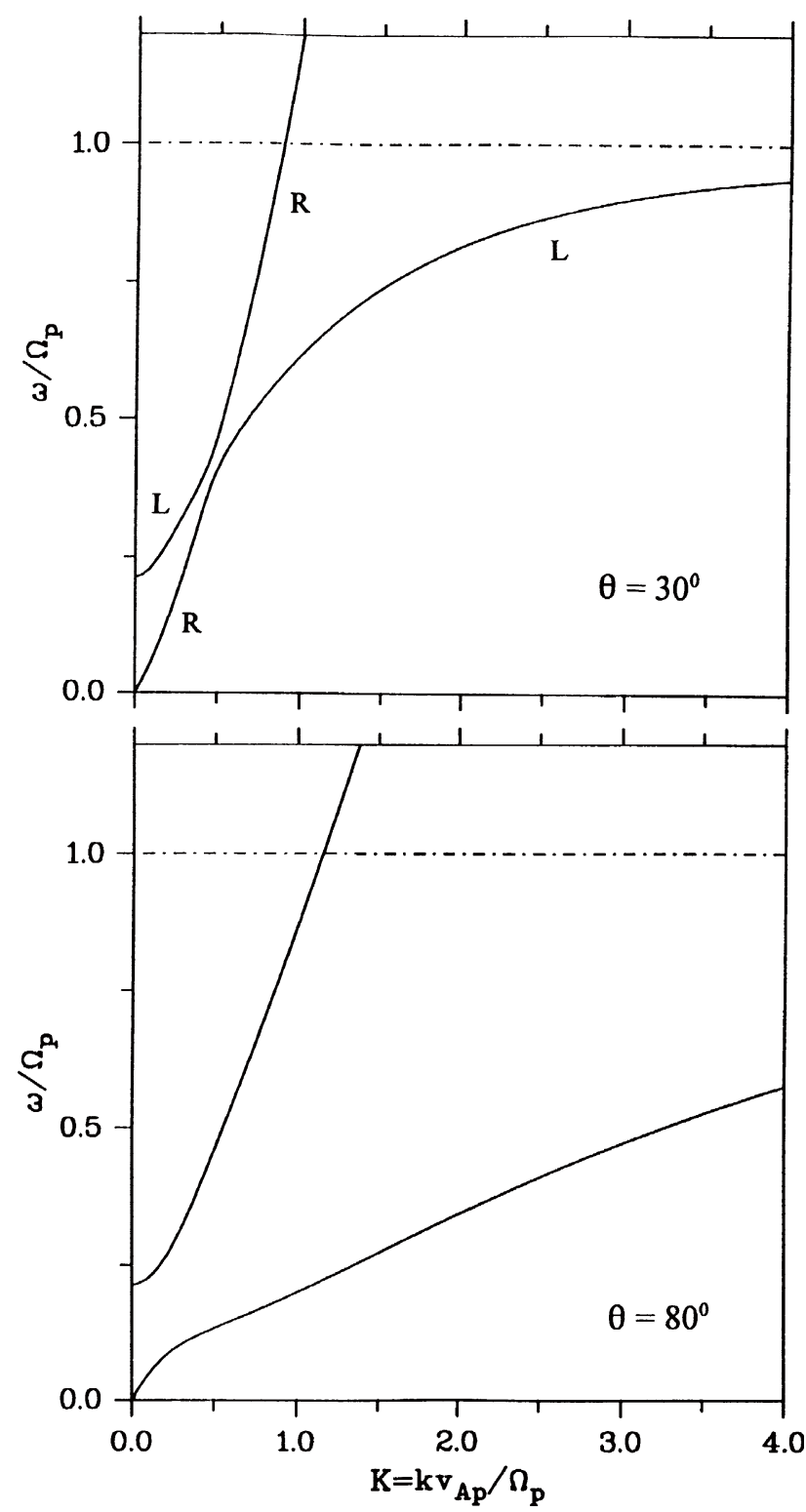

Fig. 4. Low-frequency wave dispersion in a cold bi-ion plasma (hydrogen plasma with oxygen ions) for two propagation angles $\theta$ between $\boldsymbol{k}$ and $\boldsymbol{B}\left(\mathrm{a}: \theta=30^{\circ}\right.$, b: $\theta=80^{\circ}$ ). R and L identify the polarization. The L mode which resonates at the heavy ion cyclotron frequency is not shown.

unmagnetized heavy ions transverse to the magnetic field is considered. In Fig. 5, the L/R mode is plotted separately up to large wave numbers of the whistler branch together with a beam mode. For beam velocities larger than the Alfven velocity $\left(v_{h x} \gg>v_{A}\right)$ there are two intersections between the $\mathrm{L} / \mathrm{R}$ mode and the beam dispersion: one is in the whistler branch and the other shown in the inset of Fig. 5 is near the cutoff-frequency. Accordingly, two separated unstable frequency bands may arise. Results of the numerical dispersion analysis for the upper frequency band are plotted in Fig. 6 which shows the real and imaginary parts of the frequency of the relevant wave modes versus the wave number $k$ for a beam velocity of $M_{\mathrm{A}}=\mathrm{v}_{\mathrm{hx}} / \mathrm{v}_{\mathrm{A}}=8$ and oblique propagation $\left(\theta=35^{\circ}\right)$. Figure $6(\mathrm{a})$ depicts the $\omega-k$ relation in the solar wind frame. This pattern clearly arises from the intersection of the beam mode $\left(\omega \approx k \mathrm{v}_{\mathrm{hx}} \sin \theta\right)$ with the 


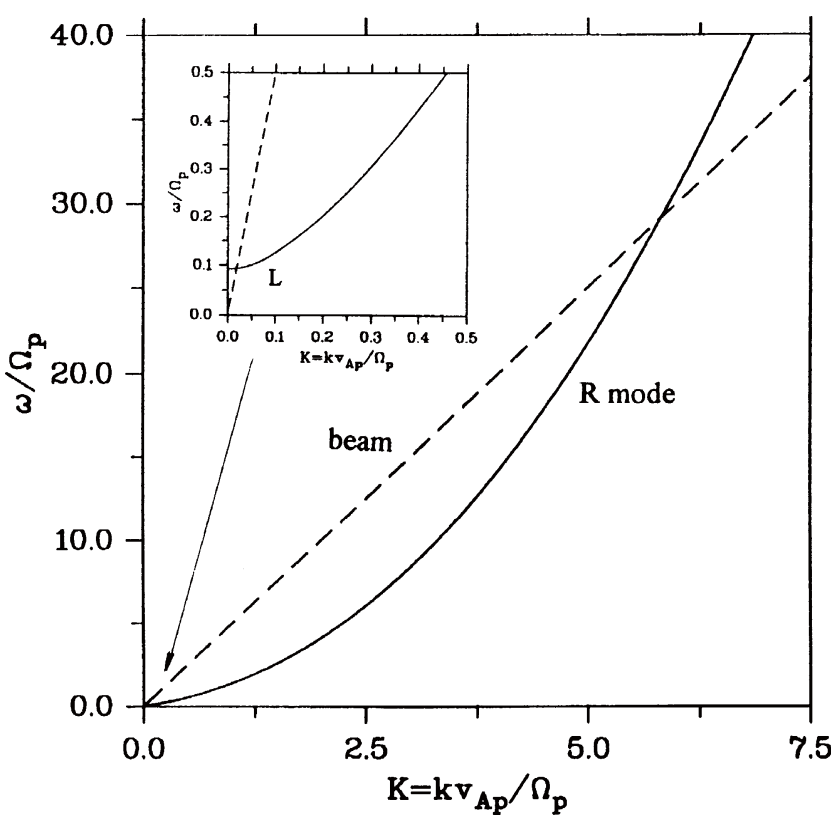

Fig. 5. The upper proton L/R mode from Fig. 4 over a broader range of frequencies up to the whistler branch. The dashed line represents a beam mode indicating possible intersections. The inset shows the wave dispersion near the cutoff frequency $\omega_{\text {cf }}$.

whistler (R) mode of the background plasma. The most unstable waves propagate oblique to the magnetic field, in contrast to several ion/ion instabilities discussed in literature which predict the most unstable mode to propagate parallel to the magnetic field. As seen in Fig. 6(c), there is a sharp threshold in the wave number $k$ for the onset of the instability, and the maximum growth rate is attained just adjacent to it. The $\omega-k$ relation of this unstable wave in the beam frame $\left(\omega^{*}=\omega-\boldsymbol{k} \cdot \mathbf{v}_{\mathrm{h}}\right)$, which is almost the spacecraft frame where the heavy ions are nearly at rest, is shown in Fig. 6(b). It is evident that the wave near maximum growth is Doppler-shifted to the proton cyclotron band, that is

$$
\omega-k \mathrm{v}_{\mathrm{hx}} \approx-\Omega_{\mathrm{p}} \text { or } \omega^{*} \approx-\Omega_{\mathrm{p}}
$$

leaving behind a left-hand polarized wave in the beam frame. Further, it is important to know how the instability varies with the propagation angle $\theta$. From the dispersion characteristics in Fig. 7 it follows that both the growth rate and the related wave number increase as the angle $\theta$ increases.

The occurrence of another instability in the lower frequency band (for the same parameters as in Fig. 6) is shown in Fig. 8. As discussed before, it arises due to the coupling between the beam and the $\mathrm{L}$ mode near the cutoff-frequency $\omega_{\mathrm{cf}}$. Doppler-shift into the beam frame brings the frequency to a fraction of the proton gyrofrequency $\left(\omega^{*} \leq 0.1 \Omega_{\mathrm{p}}\right)$. We believe that this type of beam instability is similar to that discussed in the paper by Brinca and Tsurutani (1987) where a maximum growth rate was found away from parallel propagation under the assumption that heavy (cometary) ions have large perpendicular energies, see also Brinca (1991).

A crucial problem for the physical relevance of the beamdriven electromagnetic instability is wave damping by ki-
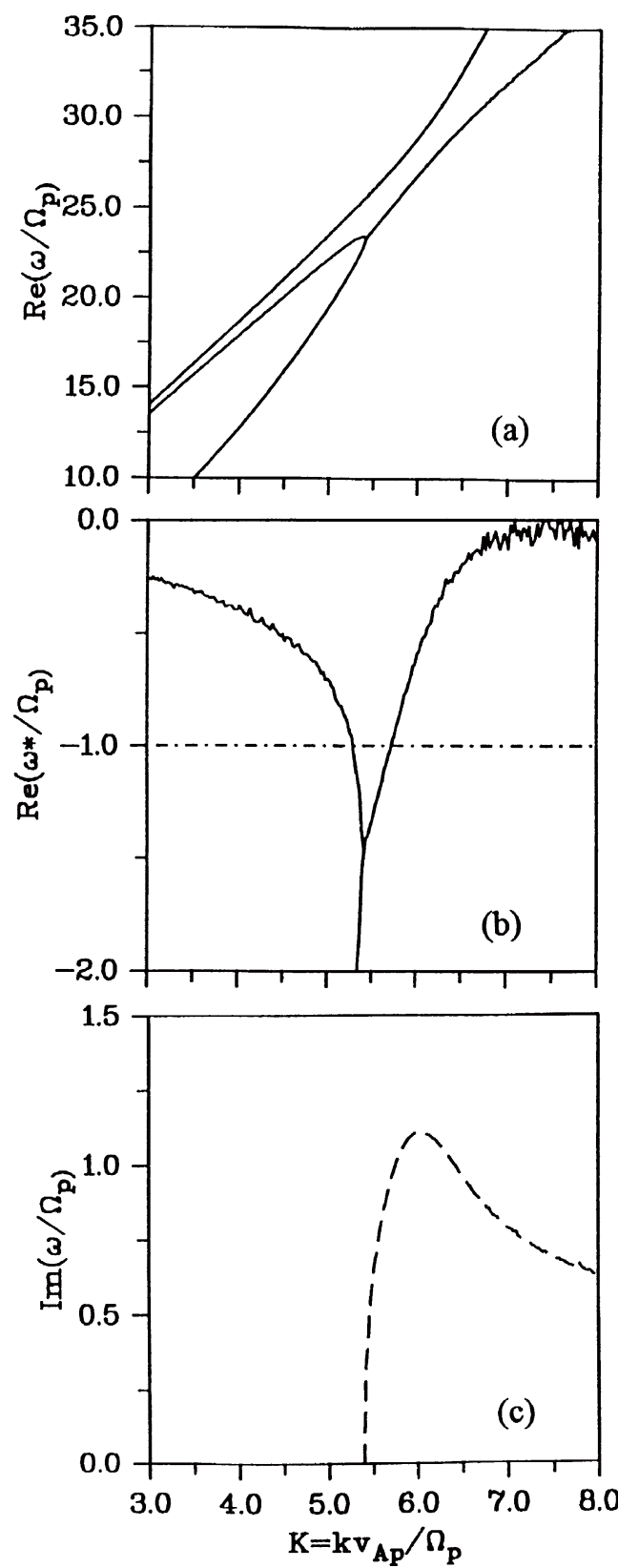

Fig. 6. Dispersion diagram for the unstable wave in the whistler branch (upper instability) driven in the solar wind by an oxygen beam $\left(M_{\mathrm{A}}=\right.$ $\left.8, n_{\mathrm{n}} / n_{\mathrm{p}}=0.05\right)$ transverse to the magnetic field. The wave propagates in an angle of $\Omega=35^{\circ}$ to the magnetic field. The top panel (a) shows the real part of the frequency (normalized to the proton cyclotron frequency $\Omega_{\mathrm{p}}$ ) versus the wave number $k$ (normalized by the proton inertial length $\mathrm{c} / \omega_{\mathrm{p}}=\mathrm{v}_{\mathrm{Ap}} / \Omega_{\mathrm{p}}$ ) in the solar wind frame. In the middle panel (b) the same relation is plotted in the beam reference frame $\left(\omega^{*}\right.$ $=\omega-\boldsymbol{k} \cdot \mathbf{v}_{\mathrm{h}}$ ). The bottom panel (c) shows the corresponding imaginary part of the frequency (growth rate) versus $k$. It is obvious, that maximum growth rate in the beam frame appears near the proton cyclotron frequency, $\omega^{*} \approx \Omega_{\mathrm{p}}$.

netic effects, which are excluded by our fluid approach. Generally, kinetic damping of cyclotron waves is caused by the resonance between the rotating electric field of the wave and the gyrating particles parallel to the magnetic field. For oblique propagation the picture becomes more complex. Generally, the damping will increase with increasing wave number. Especially, the "upper" instability in the whistler branch where $k$ is relatively large may strongly be affected 

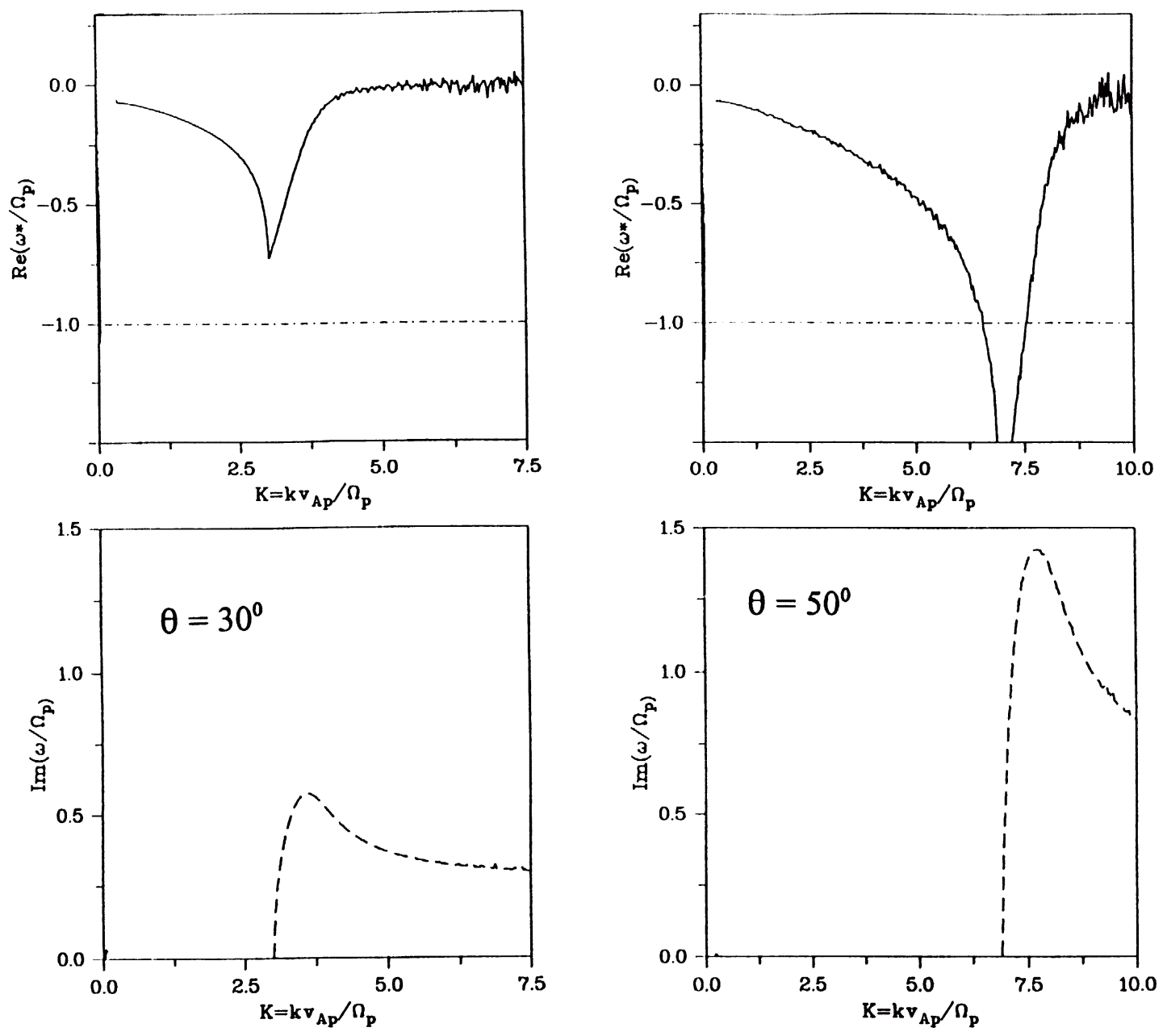

Fig. 7. Dispersion diagram of the upper unstable wave in the beam reference frame for two different angles of propagation; (a) $\theta=30^{\circ}$, (b) $\theta=50^{\circ}$. The other parameters are the same as in Fig. 6. The shift of the wave number threshold for the onset of the instability to higher $k$ and the increase of the growth rate with increasing $\theta$ are clearly seen.

by kinetic effects. A reduction of the growth rate with increasing $k$ can be expected leading to a sharper peak instead of the broad distribution in Fig. 6(c). Further, owing to the increase of $k_{\text {threshold }}$ with increasing propagation angle $\theta$, an optimum angle may result for this whistler-type instability. To come to a more quantitative picture, we looked to published results in the literature. Whistler damping for oblique propagation was studied e.g. by Tokar and Gary (1984) and Gary and Mellot (1985). Their damping rates $\gamma$ from the Vlasov theory are suitable to determine reduced growth rates by adding the kinetic damping rates to the growth rates of our beam-instability. Crucial parameters are the ratio of the electron thermal energy to the magnetic energy density $\left(\beta_{\mathrm{e}}\right)$ and the propagation angle $\theta$ (see Fig. 2 in Gary and Mellot, 1985). As an example, for $\beta_{\mathrm{e}}=0.4$ the resulting growth rate versus wave number is shown in Fig. 9. As expected, the growth rate is reduced and a much more localized peak appears compared with the case without kinetic damping. With respect to the dependence on $\theta$, Fig. 4 in Gary and Mellot (1985) exhibits the following variation: the damping rate $\gamma$ at first increases with increasing $\theta$; for $\theta$ $\geq 80^{\circ}$, however, the damping rate sharply decreases and is nearly zero at $90^{\circ}$, where the wave becomes purely compressive. This damping dependence, linearly combined with the growth rates of our upper instability, leads to the following conclusion: instead of a continuous increase of the growth rate with $\theta$, a variation with two maxima results (see also Sauer et al., 1998). The first peak arises at about $20^{\circ}-30^{\circ}$ indicating the excitation of whistler-like waves, the same as stated by Baumgärtel et al. (1998). The second, even larger peak (not discussed there) occurs at $\theta \geq 80^{\circ}$ for waves with mostly compressive magneto-acoustic signatures. A kinetic treatment of the "lower instability" in the range of small $k$ (Fig. 8) will probably bring about only weak modifications of our fluid results. In any case, a detailed kinetic dispersion analysis is needed for both cases.

\section{Discussion}

The fact that the presence of minor heavy ions in a (mostly proton) plasma flow may lead to significant changes in the spectrum of waves is known since the first studies in the sixties (e.g. Smith and Brice, 1964). In-situ measurements of the cometary environment by space missions since 1985 (ICE, VEGA/GIOTTO) had a great stimulus on this complex topic. It was found that the direction of the magnetic field relative to the solar wind is of great importance for wave modes which are excited by the pickup of heavy ions. In many papers unstable wave generation by ring-beam ion 

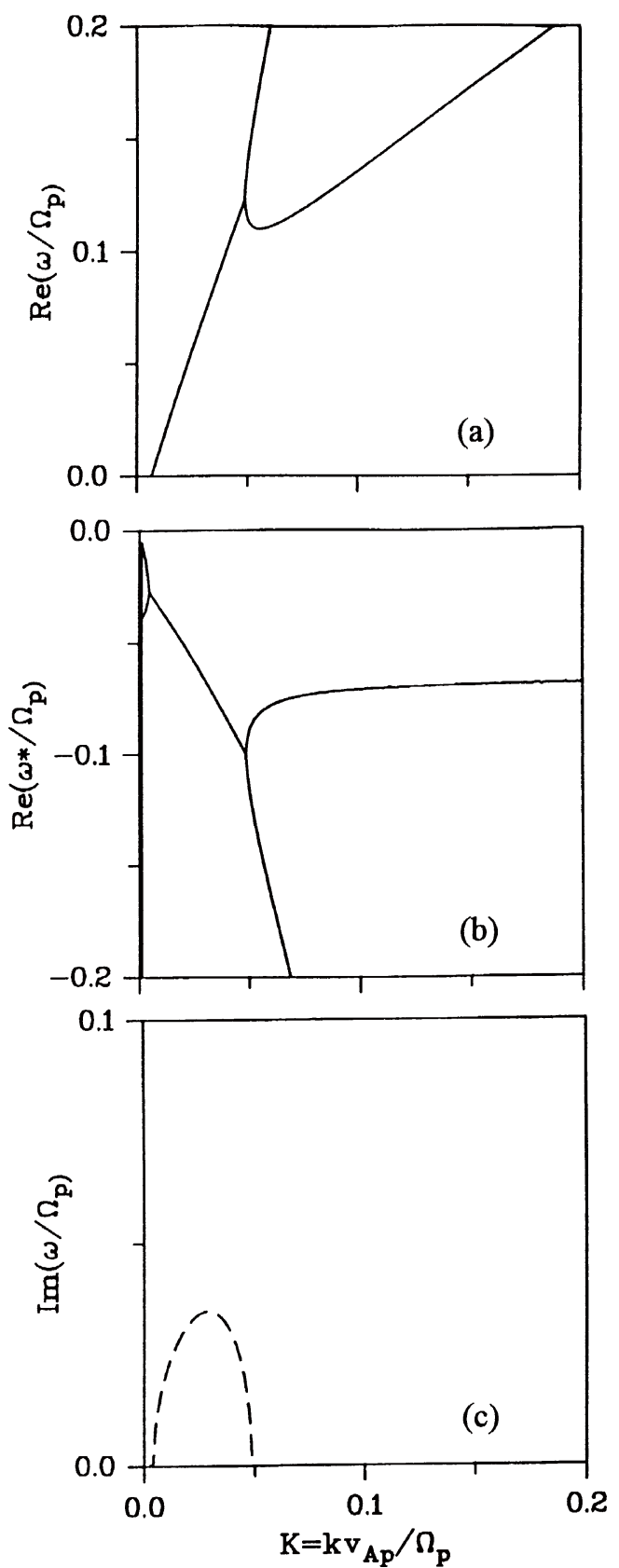

Fig. 8. Dispersion diagram for the unstable wave in the frequency branch near to the cutoff-frequency (lower instability) driven by an oxygen beam with the same parameters as in Fig. 6; (a) $\operatorname{Re}(\omega)$ in the solar wind frame, (b) $\operatorname{Re}(\omega)$ in the beam frame and (c) $\operatorname{Im}(\omega)$ over $k$.

distributions was studied. But, as discussed in the review by Tsurutani (1991) several unsolved problems still exist. A mystery, for example, is the lack of proton cyclotron waves near comets. Only magnetic pulses with a duration of the proton cyclotron period were seen.

In our present investigation which was stimulated by the observation of electromagnetic waves in the proton cyclotron band in the vicinity of Mars (as Phobos events and bow shock upstream signatures) a new type of instability in a multi-ion plasma was considered. It is caused by minor heavy ions from a localized source in a plasma flow under the assumption that the interaction region is not extended enough for the newly created ions to carry out a full gyration.
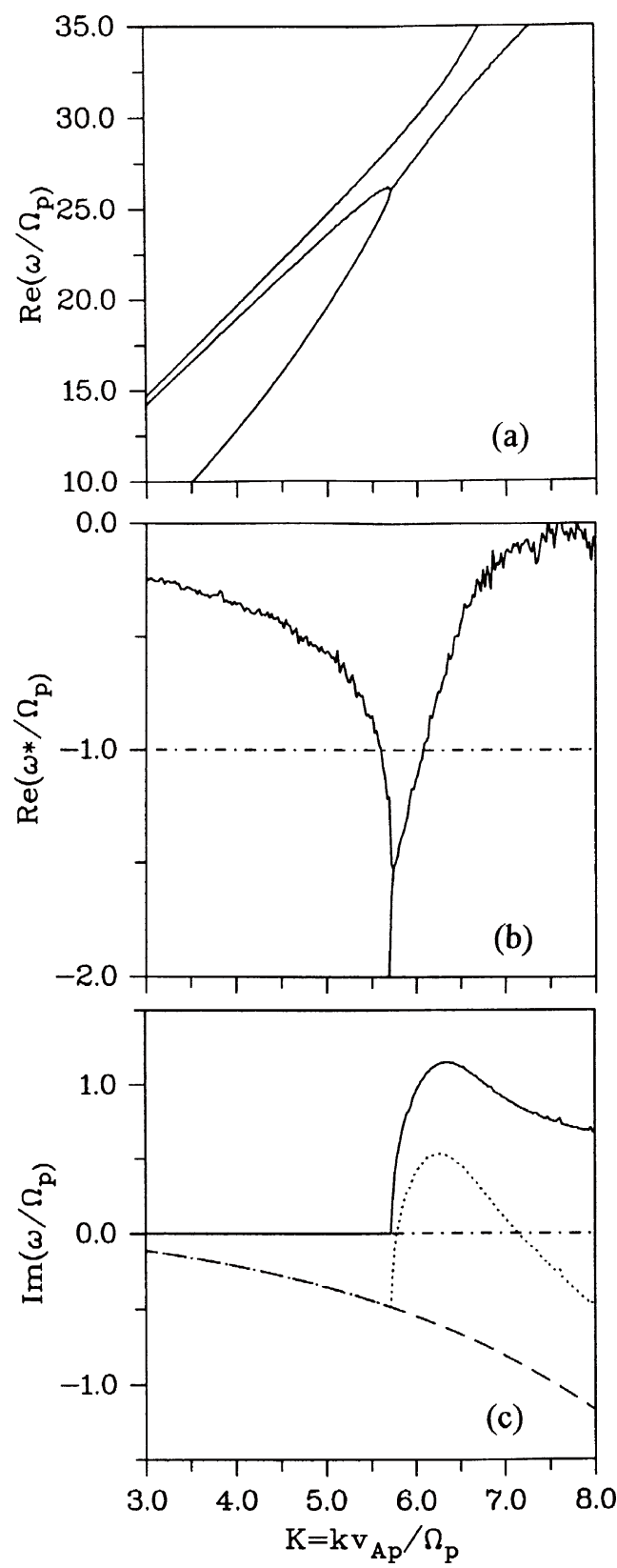

Fig. 9. The same dispersion relation as in Fig. 6, but including the effect of kinetic damping on the growth rate (bottom panel). The damping rate (dashed curve) was taken from Gary and Mellot (1985) for $\beta_{\mathrm{e}}=0.4$. The dotted curve shows the resulting dependence $\operatorname{Im}(\omega)$ versus $k$, adding the growth rate of the cold theory (solid curve) to the damping rate of the kinetic (Vlasov) theory. The maximum increment is reduced and more localized in $k$.

(Up to a certain sense, this picture contains some elements of kinetic models (Motschmann et al., 1997) in which nongyrotropic distributions are used.) Over such restricted distances the heavy ion can be considered as unmagnetized forming a beam in the magnetized proton/electron plasma (regardless of the magnetic field direction). As a result of the fluid dispersion analysis for low-frequency waves in this particular beam-plasma system, it was shown that two types of unstable electromagnetic waves can be excited which are most unstable oblique to the magnetic field: one is in the frequency branch near to the cutoff-frequency $\omega_{\mathrm{cf}}$, and the other is in the whistler/ magneto-acoustic branch. Doppler 
shift into the beam frame gives the frequency band relevant for spacecraft observations: The lower band is far below the proton cyclotron frequency $\Omega_{\mathrm{p}}$, and the upper one is, to our own surprise, closely located to $\Omega_{\mathrm{p}}$. Both bands were clearly seen in the Phobos- 2 observations upstream of the Martian bow shock, especially at the Phobos events. The growth rate of both unstable waves increases and shifts to higher wave numbers with increasing propagation angle. However, a limitation of this trend by kinetic damping appears leaving behind two optimal angles of propagation, (depending on $\beta_{\mathrm{e}}$ ) one is about $20^{\circ}-40^{\circ}$ (whistler-like waves) and the other is close to $90^{\circ}$ (compressive magneto-acoustic waves), in good agreement with the AMPTE observations (Sauer et al., 1998). The fluid approach has to be considered as a first step, having the advantage that the off-magnetic field propagation can much easier be handled than in a corresponding kinetic model. Much work remains, including more complete studies on the damping and polarization of the beam-excited waves. Finally, we want to give an outlook on an extended multi-ion beam-plasma model where the core plasma is already composed of two magnetized ion populations, protons and implanted heavy ions, which interact with an unmagnetized heavy ion beam as the third component. The splitting of heavy ions in two populations may reflect real situations near weakly outgassing comets, like GriggSkjellerup where the incoming plasma flow of protons and (in large distances) completely implanted ions interacts with newly generated cometary ions nearer to the comet which have not enough space to carry out a complete gyration. This leads to excitation of waves near the heavy ion gyrofrequency, as observed.

Acknowledgments. The authors wish to express their thanks to the International Space Science Institute (ISSI) in Bern where most of the work was done for their support of the collaboration in the framework of the Scientific Visiting Program.

\section{References}

Baumgärtel, K., K. Sauer, A. Bogdanov, E. Dubinin, and M. Dougherty, "Phobos events": signatures of solar wind dust interaction, Planet. Space Sci., 44, 589-601, 1996.

Baumgärtel, K., K. Sauer, E. Dubinin, V. Tarasov, and M. Dougherty, "Phobos events" - signatures of solar wind interaction with a gas torus?, Earth Planets Space, 1998 (in press).

Brecht, S., Solar wind proton deposition into the Martian magnetosphere, J. Geophys. Res., 102, 11,287-11,294, 1997.

Brinca, A. L., Cometary linear instabilities: From profusion to perspective, in Cometary Plasma Processes, pp. 211-221, Geophys. Monograph 61, 1991.

Brinca, A. L. and B. T. Tsurutani, Unusual characteristics of electromagnetic waves excited by cometary newborn ions with large perpendicular energies, Astron. Astrophys., 187, 311, 1987.

Dubinin, E., The Phobos and Deimos effects, Adv. Space Res., 13, 271, 1993.

Dubinin, E., R. Lundin, N. F. Pissarenko, S. V. Barabash, A. V. Zakharov, H. Koskinen, K. Schwingenschuh, and Y. Yeroshenko, Indirect evidence for a gas/dust torus along the Phobos orbit, Geophys. Res. Lett., 17, 861, 1990.

Dubinin, E., D. Obod, A. Pedersen, and R. Grard, Mass-loading asymmetry in upstream region near Mars, Geophys. Res. Lett., 21, 2769-2772, 1994.

Dubinin, E., K. Sauer, R. Lundin, K. Baumgärtel, and A. Bogdanov, Structuring of the transition region (plasma mantle) of the Martian magnetosphere, Geophys. Res. Lett., 23, 785-788, 1996.

Gary, S. P. and M. M. Mellot, Whistler damping at oblique propagation: laminar shock precursors, J. Geophys. Res., 90, 99-104, 1985.

Gomberoff, L., G. Gnavi, and F. T. Gratton, Minor heavy ion electromagnetic beam-plasma interactions in the solar wind, J. Geophys. Res., 101, 13,517-13,522, 1996.

Gurnett, D. A., R. R. Anderson, T. Z. Ma, G. Haerendel, G. Paschmann, O. Bauer, R. Treumann, H. Koons, R. H. Holzworth, and H. Lühr, Waves and electric fields associated with the first AMPTE artificial comet, J. Geophys. Res., 91, 10013-10028, 1986a.

Gurnett, D. A., T. Z. Ma, R. R. Anderson, O. Bauer, G. Haerendel, B. Häusler, G. Paschmann, R. Treumann, H. C. Koons, R. H. Holzworth, and H. Lühr, Analysis and interpretation of shocklike electrostatic noise observed during the AMPTE solar wind lithium releases, $J$. Geophys. Res., 91, 1301-1310, 1986b.

Huddleston, D. E., R. J. Strangeway, J. Warnecke, C. T. Russel, and M. G. Kivelson, Ion cyclotron waves in the Io torus during the Galileo encounter: Warm plasma dispersion analysis, Geophys. Res. Lett., 24, 2143-2146, 1997.

Ip, W.-H., On a hot oxygen corona of Mars, Icarus, 76, 135-145, 1988. Lühr, H., D. J. Southwood, N. Klöcker, M. Acuna, B. Häusler, M. W. Dunlop, W. A. Mier-Jedrzejowics, R. P. Rijnbeek, and M. Six, In situ magnetic field measurements during the AMPTE solar wind $\mathrm{Li}^{+}$ releases, J. Geophys. Res., 91, 1261-1270, 1986.

Motschmann, U., H. Kafemann, and M. Scholer, Nongyrotropy in magnetoplasmas: simulation of wave excitation and phase-space diffusion, Ann. Geophys., 15, 603-613, 1997.

Omidi, N. and D. Winske, A kinetic study of solar wind mass loading and coemtary bow shocks, J. Geophys. Res., 92, 13,409-13,426, 1987.

Papadopoulos, K., J. D. Huba, and A. T. Y. Lui, Collisionless coupling in the AMPTE artificial comet, J. Geophys. Res., 92, 47-54, 1987.

Riedler et al., Magnetic fields near Mars: First results of the Phobos mission, Nature, 341, 604-607, 1989.

Russel, C. T., J. G. Luhmann, K. Schwingenschuh, W. Riedler, and Y. Yeroshenko, Upstream waves at Mars: Phobos observations, Geophys. Res. Lett., 17, 897-900, 1990.

Sauer, K., T. Roatsch, U. Motschmann, K. Schwingenschuh, R. Lundin, H. Rosenbauer, and S. Livi, Observations of the plasma boundaries and phenomena around Mars with Phobos 2, J. Geophys. Res., 97, 6227$6233,1992$.

Sauer, K., K. Baumgärtel, and U. Motschmann, Phobos events as precursors of solar wind-dust interaction, Geophys. Res. Lett., 20, 165-168, 1993.

Sauer, K., A. Bogdanov, and K. Baumgärtel, Evidence of an ion composition boundary (protonopause) in bi-ion fluid simulations of solar wind mass-loading, Geophys. Res. Lett., 21, 2255-2258, 1994.

Sauer, K., E. Dubinin, K. Baumgärtel, and A. Bogdanov, Deimos-an obstacle to the solar wind, Science, 269, 1075-1078, 1995.

Sauer, K., A. Bogdanov, K. Baumgärtel, and E. Dubinin, Plasma environment of comet Wirtanen during its low-activity stage, Planet. Space Sci., 44, 715-729, 1996.

Sauer, K., E. Dubinin, and K. Baumgärtel, Bi-ion structuring in the magnetosheath of Mars: Theoretical modelling, Adv. Space Res., 20, 137-142, 1997a.

Sauer, K., A. Lipatov, K. Baumgärtel, and E. Dubinin, Solar wind-Pluto interaction revised, Adv. Space Res., 20, 295-299, 1997 b.

Sauer, K., E. Dubinin, M. Dunlop, and V. Tarasov, Low-frequency electromagnetic waves near the proton cyclotron frequency at the AMPTE Ba release: Relevance to weak comets and Mars, Geophys. Res. Lett., 1998 (submitted).

Smith, R. L. and N. Brice, Propagation in multi-component plasmas, J. Geophys. Res., 69, 5029, 1964.

Stix, T. H., Waves in Plasmas, p. 7, American Institute of Physics, New York, 1992.

Tarasov, V., E. Dubinin, S. Perraut, K. Sauer, and A. Skalsky, Wavelet application to the magnetic field turbulence in the upstream of the Martian bow shock, Earth Planets Space, 1998 (submitted).

Tokar, R. L. and P. S. Gary, The whistler mode in a Vlasov plasma, Phys. Fluids, 28, 1063-1069, 1984.

Tsurutani, B. T., Comets: a laboratory for plasma waves and instabilities, in Cometary Plasma Processes, pp. 189-209, Geophysical Monograph 61, AGU, 1991.

Tsurutani, B. T. and E. J. Smith, Hydromagnetic waves and instabilities associated with cometray-ion pickup: ICE observations, Geophys. Res. 
Lett., 13, 263-266, 1986.

Verigin, M. I., K. I. Gringauz, G. A. Kotova, N. M. Shutte, H. Rosenbauer, S. Livi, A. K. Richter, W. Riedler, K. Schwingenschuh, and K. Szegö, On the problem of the Martian atmosphere dissipation: Phobos-2

TAUS spectrometer results, J. Geophys. Res., 96, 19,315-19,320, 1991 Warnecke, J., M. G. Kivelson, K. K. Khurana, D. E. Huddleston, and C.

T. Russel, Ion cyclotron waves observed at Galileo's Io encounter: Implications for neutral cloud distribution and plasma composition, Geophys. Res. Lett., 24, 2139-2142, 1997.
Young, D. T., S. Perrault, A. Roux, C. de Villedary, R. Gendrin, A. Korth, K. Kremser, and D. Jones, Wave-particle interactions near $\Omega_{\mathrm{He}}{ }^{+}$observed on GEOS1 and 2, J. Geophys. Res., 86, 6755, 1981.

K. Sauer (e-mail: sauer@ linmpi.mpg.de), K. Baumgärtel (e-mail: kbaumgaertel@aip.de),E.Dubinin(e-mail:dubinin@helene.mpae. gwdg.de), and V. Tarasov 